\title{
Return to Play in Adolescent Athletes With Symptomatic Spondylolysis Without Listhesis: A Meta-Analysis
}

\author{
Samuel C. Overley, MD', Steven J. McAnany, MD', \\ Steve Andelman, MD', Jun Kim, MD', Robert K. Merrill, BS', \\ Samuel K. Cho, MD', Sheeraz A. Qureshi, MBA, MD', \\ and Andrew C. Hecht, MD'
}

\begin{abstract}
Study Design: Meta-analysis.

Objectives: To assess return to play (RTP) rates in adolescent athletes with lumbar spondylolysis without spondylolisthesis treated conservatively or operatively.

Methods: A review of Medline, EMBASE, and Cochrane Reviews was performed. The pooled results were performed by calculating the effect size based on the logit event rate. Studies were weighted by the inverse of the variance. Confidence intervals were reported at $95 \%$. Heterogeneity was assessed using the $Q$ statistic and $I^{2}$ value.

Results: The initial literature search resulted in 724 articles, of which 29 were deemed relevant on abstract review. Overall, I I studies provided data for $\mathbf{3 7 6}$ patients with a pars interarticularis defect. Return to athletic competition, based on logit event rate, was found to be statistically favored after both nonoperative and operative treatment (92.2\% vs $90.3 \%)$. There was no heterogeneity noted among the studies reporting nonoperative treatment ( $Q$ value of 4.99 and $I^{2}$ value of 0 ). There was mild heterogeneity within the operative studies ( $Q$ value of 3.54 and $l^{2}$ value of 15.18 ).

Conclusions: Adolescent athletes RTP $92.2 \%$ of the time with nonoperative management, compared with $90.3 \%$ when treated operatively, though both treatment groups strongly favor RTP. As this is the first study to pool results of all relevant literature, it provides strong evidence to guide decision making and help manage expectations in this unique patient population.
\end{abstract}

\section{Keywords}

return to play, meta-analysis, adolescent athletes, spondylolysis

\section{Introduction}

Management of symptomatic lumbar spondylolysis without listhesis in adolescent athletes represents a unique challenge for the treating physician. In the general population, the majority of cases can be successfully treated with rest and conservative management. Surgical intervention is indicated only for those few individuals who have failed a prolonged ( $>6$ months) trial of conservative management. A variety of surgical techniques have been described, although most involve either direct repair of the pars interarticularis with screw fixation and bone grafting as described by Buck, ${ }^{1}$ or indirect reduction and compression achieved through a tension wire construct as described by Nicol and Scott. ${ }^{2}$
Elite-level adolescent athletes, especially those who participate in sports requiring repetitive hyperextension maneuvers, place significantly greater biomechanical demands on the lumbar spine. Furthermore, there are frequently external pressures to ensure that such athletes return to preinjury levels of activity

\footnotetext{
${ }^{\prime}$ Icahn School of Medicine at Mount Sinai, New York, NY, USA

Corresponding Author:

Andrew C. Hecht, Department of Orthopedic Surgery, Icahn School of Medicine at Mount Sinai, 5 East 98th Street, 4th Floor, Box I I88, New York, NY 10029, USA.

Email: Andrew.hecht@mountsinai.org
} 
as soon as possible. Although a trial of conservative management followed by surgical intervention for persistent pain has been shown to be very effective for recreational-level athletes, less is known regarding the utility of this algorithm in higher level athletes. Recently, a number of studies have reported on the rate of return to play (RTP) after both conservative and surgical management of isthmic spondylolysis without listhesis in high-level athletes. In this meta-analysis, we evaluate the recent published literature regarding the efficacy of conservative and operative management with regard to rate of RTP.

\section{Materials and Methods}

\section{Inclusion and Exclusion Criteria and Review Procedure}

A systematic computerized literature search was performed using PubMed, Cochrane Database of Systematic Reviews, and EMBASE. The electronic databases were searched from January 1990 to December 2014. Searches were performed from Medical Subject Headings (MeSH) used by the National Library of Medicine. Specifically, MeSH terms "spondylolysis," "athlete," and "sport" were used. The review procedure was conducted in accordance with PRISMA guidelines. A flow diagram of the systemic review is presented in Figure 1. Inclusion criteria for the meta-analysis included the following: age $<25$ years, radiologic pars defect without evidence of spondylolisthesis, athletic participation, documentation of return to sport activity, method of operative or nonoperative treatment, and a methodological quality score greater than or equal to 10 . The abstracts for each article were reviewed by 2 independent authors to assess for inclusion in the meta-analysis. The authors jointly reviewed the full texts of the articles meeting the inclusion criteria for the study. A third author was available in the event of a discrepancy between the 2 reviewers, and a consensus was reached after review. The methodological quality of each study was appraised in accordance with the Downs and Black checklist. ${ }^{3}$

\section{Data Extraction}

A database was created from the included studies with the following categories: study ID to include author, journal, and year of publication; reference; study type and level of evidence; number of patients; sport played; patient age; length of follow-up; operative or nonoperative definitive treatment; operative levels; surgical indication; duration between surgery to RTP; and clinical success. The primary outcome variable was ability to RTP, defined as returning to sport and logging regular season playing time in at least one game. Success was defined by an ability to RTP, whereas failure was defined by an inability to RTP.

\section{Methodological Quality Assessment}

Methodological quality assessment was accomplished using the Downs and Black checklist. It is a checklist that culminates in a total score that is directly proportional to the quality of the

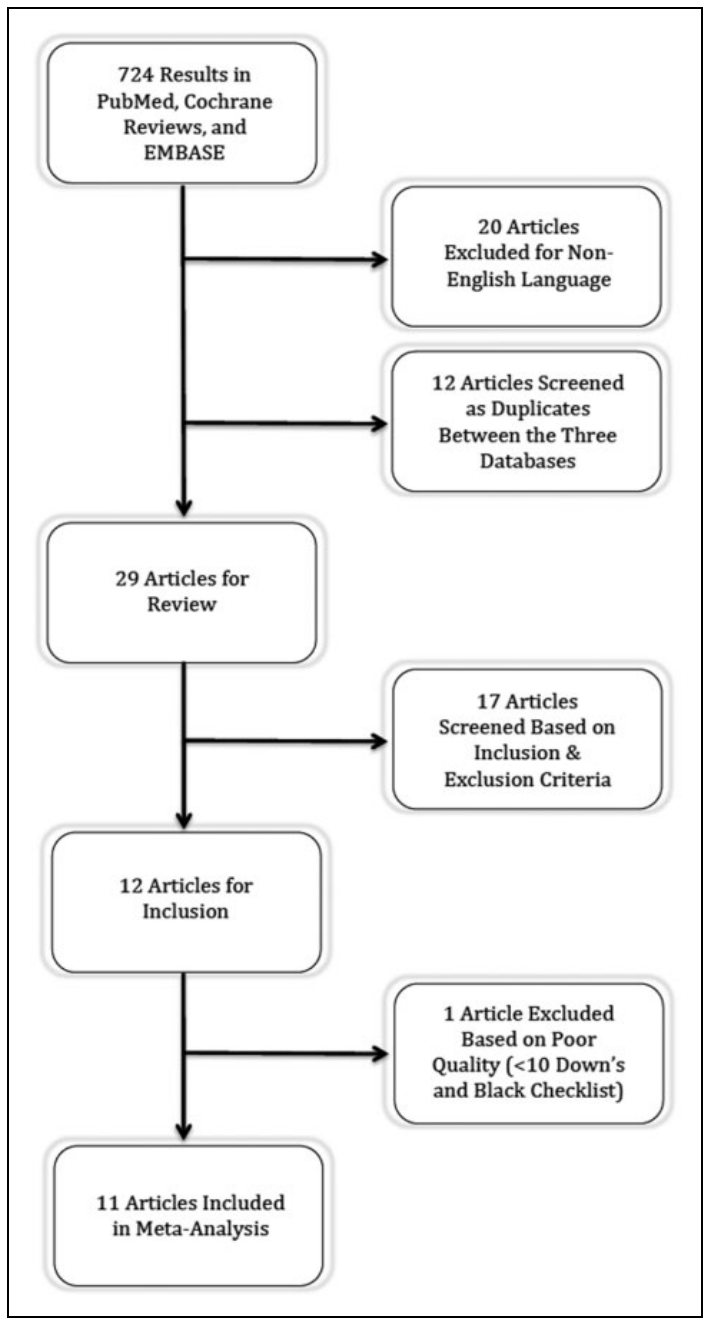

Figure I. PRISMA flow chart.

study. The total cumulative score is composed of a profile that measures quality of reporting, internal validity (bias and confounding), and external validity. According to Downs et al, the performance results of the checklist showed a high internal consistency $(\mathrm{KR}-20=0.91)$, test-retest score $(r=.90)$, and interrater reliability $(r=.80)$. The checklist consists of 27 items for which an answer "yes" correlates with a score of " 1 " and an answer "no" correlates with a score of " 0 ." Notably, we used a modified Black and Downs checklist in which item 27 was shifted to a binary answer system. One point was awarded if a power or sample size calculation was present. This method produced a maximum score of 28 .

\section{Meta-Analysis}

Pooling of the results was performed by calculating the effect size based on the logit event rate (a statistical extrapolation of raw data proportions) using Comprehensive Meta Analysis, version 2.2.050 (Biostat, Englewood, NJ). The studies were weighted in the meta-analysis by the inverse of the variance, which included both within and between study errors. The effect size and confidence intervals (CIs) were reported using 
a Forest plot. Confidence intervals were reported at a $95 \%$ level. A $P$ value of .05 was set for significance. Heterogeneity was assessed using the $Q$ statistic and $I^{2}$, where $I^{2}$ is the estimate of the percentage of error due to between-study variation. $I^{2}$ values below $25 \%$ generally indicate consistent results and homogeneous studies. A priori, a random effects model was selected. A sensitivity analysis was performed by varying the assumptions used in the meta-analysis, and also through single elimination of the studies to assess for significance. A funnel plot was assessed for determination of publication bias.

\section{Results}

\section{Systematic Review}

The initial PubMed, Cochrane Review, and EMBASE search resulted in 724 articles. After 2-reviewer assessment of abstracts, 29 articles were identified as requiring further manuscript review. Eighteen articles were excluded based on inclusion criteria and quality cutoff $(<10)$, leaving a total of 11 studies included in the meta-analysis.

Seven studies reported exclusively on nonoperative treatment for young athletes with symptomatic spondylolysis without listhesis, while 4 studies examined similar patients treated operatively. There were a total of 245 patients in the nonoperative treatment group, with an average age of 18.1 (7-31) years, average follow-up of 29 (3-120) months, and average RTP timing after treatment initiation of 5.9 months. The operative arm consisted of 74 patients with an average age of 19 (13-37) years, average follow-up of 38.4 (12-135) months, and average RTP time after surgery of 6.5 months. These results are summarized in Table 1.

\section{Quality Assessment of Included Studies}

The quality index score of our 11 studies ranged from 11 to 18 . We calculated an average score of 14 and a standard deviation of 1.45 . We defined a higher quality study as 15 to 18 , a moderate quality study as 12 to 15 , and a poorer quality study as 8 to 12 . The quality scores for each article are delineated in Table 1 .

\section{Summary of Investigations}

Eleven studies were reviewed for this meta-analysis, 7 that reported outcomes after conservative management and 4 that reported outcomes after surgical intervention. Multiple surgical techniques were described, most of which were variations of either direct pars interarticularis screw fixation or utilization of a tension-band wire construct. All instances of surgical intervention were performed after a failed period of conservative management.

Donaldson studied 11 elite-level hockey players with lumbar spondylolysis without listhesis treated conservatively with rest a physical therapy. Of the 11 players with radiographically confirmed spondylolysis at an average age of 16.2 years, 10
(91\%) were able to return to preinjury level of play at a mean of 8 (6-12) weeks. ${ }^{4}$

Ruiz-Cotorro followed 66 elite-level tennis athletes with spondylosis without spondylolisthesis treated conservatively with rest followed by physical therapy. The average age of the 66 athletes was 14.8 (12-21) years. The authors reported that all $66(100 \%)$ were able to RTP at a mean of $3.5(0.5-5.5)$ months. ${ }^{5}$

Sys et al studied 28 elite-level athletes with lumbar spondylosis without listhesis, the majority of whom were soccer players. The average age of the athletes at diagnosis was 17.2 (12-27) years. After a period of rest and utilization of the Boston Overlap Brace followed by physical therapy, 25 of $28(89 \%)$ were able to achieve RTP at an elite level by an average of 22 weeks. $^{6}$

Jackson et al followed a group of 25 mixed athletes with radiographically confirmed spondylolysis without listhesis, of which 10 were lost to follow-up and not included in this metaanalysis. Of the remaining 15 young athletes, reported to play soccer, basketball, football, or gymnastics, $12(80 \%)$ were able to RTP at an average of 7.3 months after a period of rest alone. The average age at diagnosis in this cohort was 15.5 (7-20) years. ${ }^{7}$

In the largest study of its kind, Iwamoto followed a cohort of 191 elite adolescent athletes diagnosed with spondylolysis without spondylolisthesis. Of the 191 athletes, 87 were found to have evidence of degenerative disc disease and were subsequently excluded from the study. The remaining 104 patients had an average age of 20.7 (12-60) years. All patients were treated with a lumbosacral orthosis and rest. Ninety-nine of 104 (95\%) young athletes were able to RTP at an average of 5.4 (1-11) months. Of the 5 athletes that failed to RTP, the following etiologies were cited: spinal surgery to relieve low back pain, retirement from sporting activity in a 60-year-old athlete, low sporting activity with no organized sporting event, and, in 2 cases, severe subjective low back pain. ${ }^{8}$

Looking at outcomes after operative intervention, Nozawa reported on 20 athletes who underwent placement of a tension wire construct for fixation of spondylolysis without listhesis in patients that failed prior nonoperative treatment. The average age of the cohort at time of surgery was 23.7 (12-37) years, and the average follow-up period was 41.5 (16-103) months. The authors found that $18(90 \%)$ were able to RTP and all 20 patients demonstrated radiographic union of the pars defect at final follow-up, though 3 cases were complicated by wire breakage with no apparent clinical consequences. ${ }^{9}$

Menga et al followed 31 patients, 25 of whom were identified as competitive athletes, who underwent direct pars screw fixation after failed nonoperative management. The athletes, whose average age was 16 (10-37) years, were followed for a minimum of 2 years with a mean of 60 (24-135) months. The authors reported that 19 of $25(76 \%)$ achieved clinical success and were able to RTP by an average of 6 (3-10) months. This study yielded a rather high complication rate with 1 patient requiring L5-S1 fusion for recurrent pain, 2 patients sustaining screw breakage, and 1 case of infection requiring irrigation and debridement. ${ }^{10}$ 


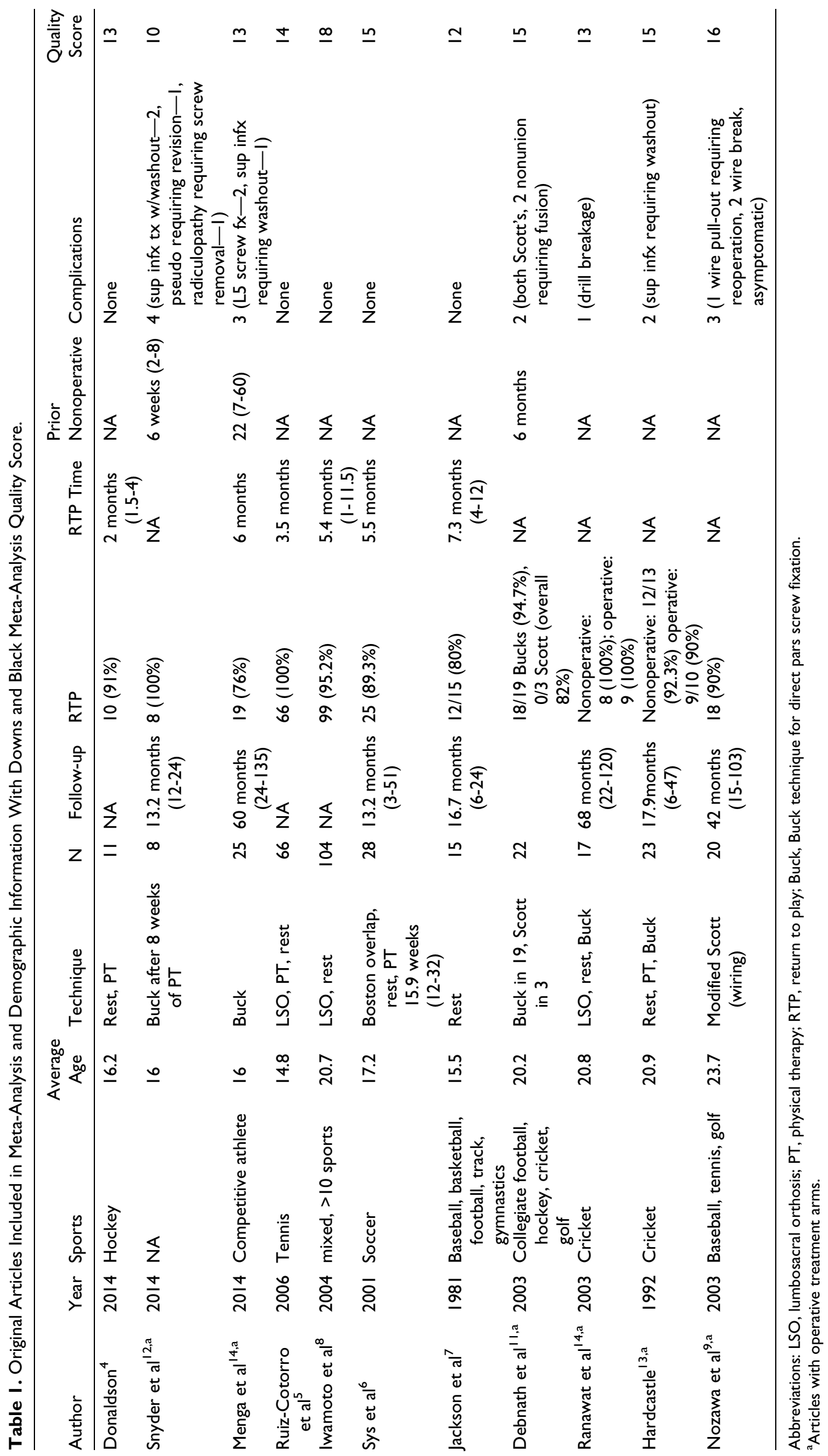




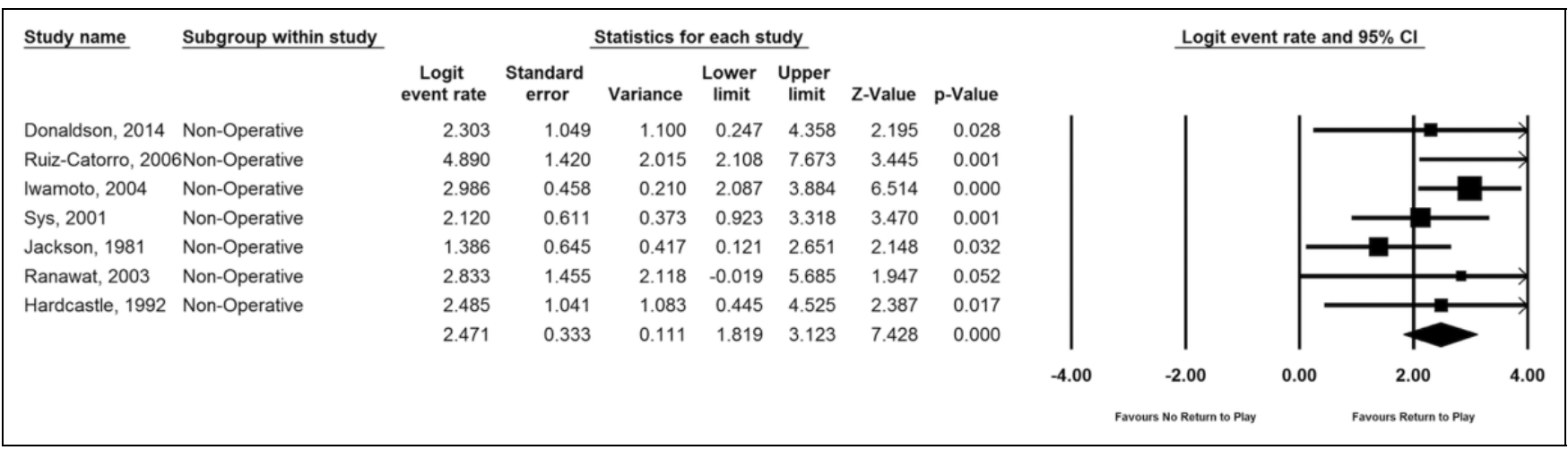

Figure 2. Forest plot of the logit event rate for return to play following nonoperative management.

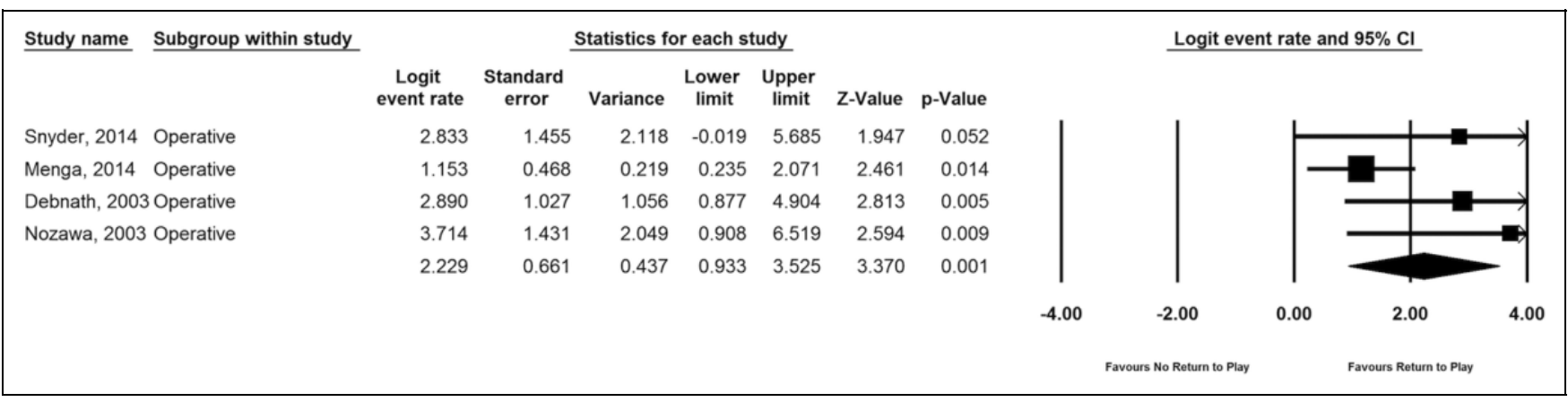

Figure 3. Forest plot of the logit event rate for return to play following operative management.

Debnath et al reported on a cohort of 22 elite-level college athletes with spondylolysis without listhesis utilizing 2 different direct pars repair techniques. The mean age of the athletes at time of surgery was 20.2 (15-34) years. Nineteen patients underwent direct pars screw fixation and 3 were treated with tension band wiring. They reported that 18 of $19(95 \%)$ patients who underwent direct screw fixation returned to play at an average of 7 (4-10) months after surgery, while none of those patients who underwent placement of a tension band wire construct were able to RTP. ${ }^{11}$

Snyder et al followed 8 athletes with spondylolysis without listhesis who failed conservative management and subsequently underwent direct screw fixation supplemented with off-label usage of rhBMP-2 as a fusion adjunct. The average age at time of surgery was 16 (11-30) years, and average follow-up was 13.2 (12-24) months. The authors reported that all 8 (100\%) were able to RTP by 6 months and experienced no perioperative or remote complications. ${ }^{12}$ Hardcastle followed 23 elite-level cricket athletes with spondylolysis without listhesis, electing to operate on only those who failed conservative management. ${ }^{13}$ Twelve of the patients were able to RTP with rest and physical therapy and one retired from sport for unrelated reasons. The remaining 10 underwent direct pars screw fixation. The average age of the patients at time of surgery was $20.9(15-25)$ years, with an average follow-up period of 17.9 (6-47) months. Of the 10 operative cases, $9(90 \%)$ were able to return to competitive play. ${ }^{13}$

Similarly, Ranawat et al followed a group of elite cricket athletes with spondylolysis without listhesis, operating on those who failed conservative management. Nine patients who failed conservative management underwent direct pars screw fixation. The average age of the cohort was 20.8 (18-31) years, and patients were followed for an average of 68 (22-120) months. All 9 surgical patients $(100 \%)$ returned to competitive play. One complication of intraoperative drill breakage without long-term sequelae was reported. $^{14}$

\section{Meta-analysis Results}

Clinical Results. Return to athletic competition was found to be statistically favored after nonoperative treatment or operative treatment $(92.2 \%$ vs $90.3 \%)$, with $P$ values $<.0001$ and $<.001$, respectively. The logit event rate of nonoperative care was calculated to be 2.47 ( $\mathrm{CI}=1.82-3.12$; Figure 2$)$. This pooled logit event rate was found to be statistically in favor of returning to sporting activity $(P<.0001)$. There was no heterogeneity noted among the studies reporting nonoperative treatment with a $Q$ value of 4.99 and $I^{2}$ value of $0 \%$. The logit event rate for operative treatment was calculated to be $2.23(\mathrm{CI}=0.93-3.53$; Figure 3). This pooled logit event rate was also found to be statistically in favor of returning to sporting activity $(P<.001)$. There was mild heterogeneity within the operative studies with $Q$ value of 3.54 and $I^{2}$ value of $15.18 \%$.

Sensitivity Analysis and Publication Bias. Single elimination of each study did not affect the overall results of the analysis. Single 


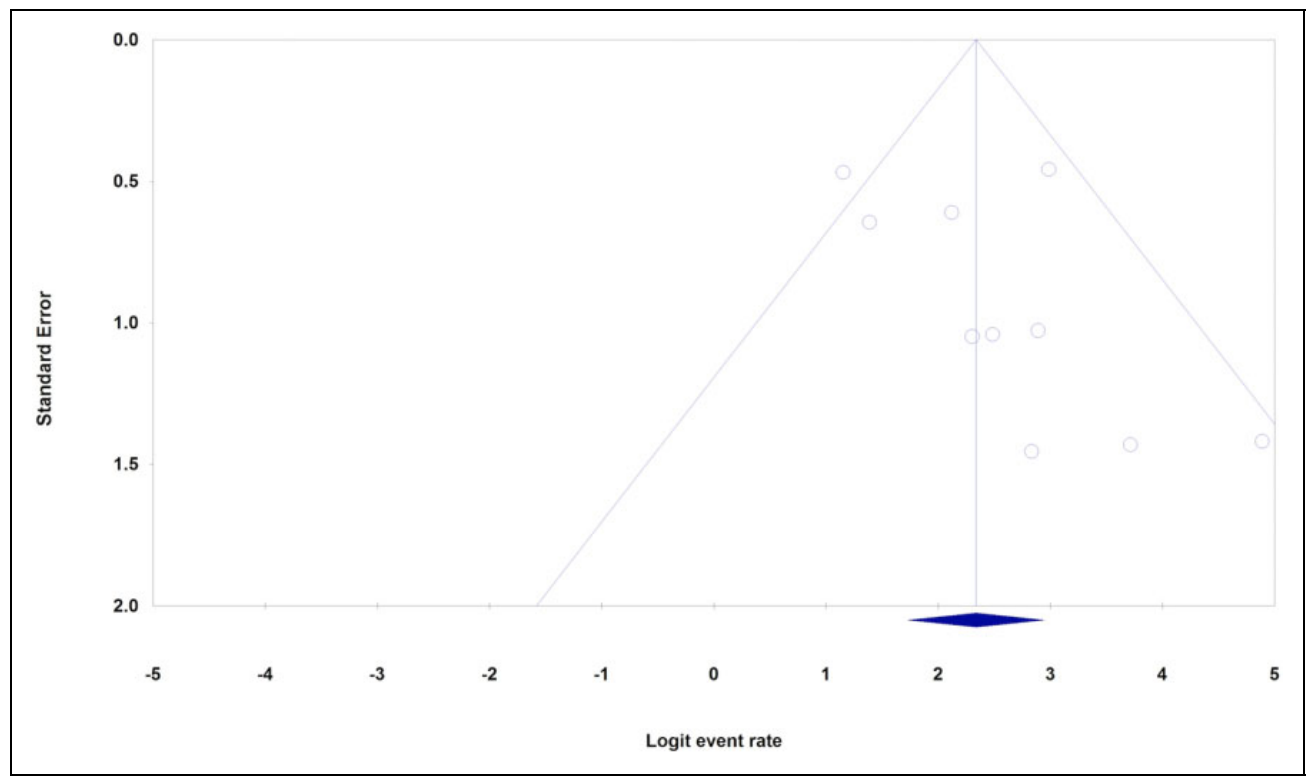

Figure 4. Funnel plot depicting selection bias.

removal of each study resulted in $P<.05$, which validates the results of the model.

Assessment of the funnel plot demonstrated symmetry about the mean logit event rate indicating a lack of publication bias (Figure 4).

\section{Discussion}

Back pain due to spondylolysis without listhesis in the adolescent athlete is a relatively common problem. Epidemiological studies have demonstrated an overall incidence of $3 \%$ to $6 \%$ in the general population, while the young athlete has been shown to carry a much higher incidence of $15 \% .{ }^{11,15,16}$ Nonsurgical management is the mainstay of treatment for patients with a symptomatic spondylolysis. ${ }^{17-23}$ However, in the sidelined adolescent elite athlete, conflicting motives exists: the desire for expeditious RTP as well as the need to heal the pain generating pars defect, which necessitates a hiatus from sporting activity. These often conflicting goals have led to advances in the surgical treatment of symptomatic spondylolysis in those who fail conservative treatment in this demographic.

Young athletes may have a lower threshold to complete lengthy periods of rest from activity, physical therapy, and core strengthening due to their desire to RTP quickly. In order to more expeditiously RTP at a high level of performance, the athlete and/or parents of the athlete may urge surgeons to pursue a more aggressive approach leading to an earlier abandonment of nonoperative management, and perhaps even definitive surgery. In 1970, Buck published seminal work describing intralaminar screw fixation and autografting of the pars defect, leading to a high rate of healing in patients who failed lengthy conservative treatment. ${ }^{1}$ This work promoted further innovation and the development of several variations of fixation methods utilizing wires, hooks, and pedicle screw instrumentation. ${ }^{2,24,25}$
With any surgical intervention comes an obligatory risk for complications, which is the salient point to consider when interpreting this meta-analysis. Although both nonsurgical and surgical treatments of young athletes with a symptomatic spondylolysis without listhesis demonstrated high levels of RTP at $92.2 \%$ and $90.3 \%$, respectively, surgery is not without cost. The pooled studies of operative treatment consisted of 74 patients, of which 15 experienced perioperative complications. These complications are further described in Table 1. With such a high complication rate, one must carefully consider the risk versus benefit profile. An algorithm consisting of an exhaustive and lengthy trial of nonoperative management prior to any surgical consideration is prudent. However, when analyzing results of this meta-analysis, the possibility of selection bias in the operative arms must be considered as all patients that received surgical intervention had failed prior nonoperative management as part of their treatment.

The use of meta-analyses allows for the pooling of data from multiple studies to evaluate whether there is a significant effect and, if so, the magnitude of the effect. Furthermore, the metaanalysis allows for assessment of the heterogeneity, or variability within the studies, which further validates the effect size. In this study, we selected a priori a random-effects model. Unlike a fixed-effects model, the random-effects model posits that the true effect may vary from study to study.

In reference to the aim of this particular study, determining RTP in young athletes with symptomatic spondylolysis without listhesis, a meta-analysis is an ideal method of analyzing the current literature comprehensively. With such a definitive outcome variable as RTP, heterogeneity among the different studies used in the analysis is unlikely. This is true in our study, which boasts a very low $I^{2}$ value for operative management and an $I^{2}$ value of zero for nonoperative treatment, indicating no heterogeneity between studies. By combining a comprehensive 
search of the English language literature and evaluating studies with little to no heterogeneity and a definitive outcome measure, results of this study can be utilized by surgeons counseling young athletes with a symptomatic spondylolysis. Surgeons may use the data presented here to guide young athletes in choosing appropriate treatment options and managing expectations with tangible values for RTP rates.

This study has several limitations, as with most meta-analyses, primarily due to low-powered studies from which the pooled results were extracted. We were not able to illustrate any favorability between the different operative fixation methods due to inadequate power. While gross inspection of the data seems to trend toward higher RTP rates with Buck's method or screw-hook constructs, we cannot comment on this with any degree of certainty. We were also unable to isolate and compare RTP rates for patients based on vertebral level involved and/or bilaterality, or stage of the pars defect. Significant selection bias may also be introduced into this study as the included studies utilized differing surgical indications as well as inconsistencies in timing and protocol of bracing and physical therapy during conservative management. Also absent in this study is any comparison of performance parameters pre- and posttreatment. This is attributed to a lack of any standardized metric for which to assess performance across a wide range of sporting events and level of competition. Additionally, true performance, even among a single sport, is difficult to define and near impossible to quantify.

\section{Conclusion}

Low back pain in the adolescent athlete is a common problem. When the etiology is spondylolysis without listhesis, several treatment options exist. Nonoperative treatment consisting of activity restriction, rest, and physical therapy with or without adjunctive bracing is the gold standard; however, not all pars defects heal with this management and some patients remain symptomatic. These patients may benefit from one of several direct pars repair techniques. The results of this study suggest that both nonoperative and operative treatments for spondylolysis results in high rates of RTP, though it should be emphasized that all patients in all included studies did undergo primary nonoperative treatment. Surgeons should employ nonoperative treatment in young athletes with surgical intervention reserved only for those who fail a lengthy dedicated course of activity restriction and physical therapy.

\section{Authors' Note}

The article does not contain information about medical device(s)/ $\operatorname{drug}(\mathrm{s})$.

\section{Declaration of Conflicting Interests}

The author(s) declared no potential conflicts of interest with respect to the research, authorship, and/or publication of this article.

\section{Funding}

The author(s) received no financial support for the research, authorship, and/or publication of this article.

\section{References}

1. Buck JE. Direct repair of the defect in spondylolisthesis. Preliminary report. J Bone Joint Surg Br. 1970;52:432-437.

2. Nicol RO, Scott JH. Lytic spondylolysis. Repair by wiring. Spine (Phila Pa 1976). 1986;11:1027-1030.

3. Downs SH, Black N. The feasibility of creating a checklist for the assessment of the methodological quality both of randomised and non-randomised studies of health care interventions. J Epidemiol Community Health. 1998;52:377-384.

4. Donaldson LD. Spondylolysis in elite junior-level ice hockey players. Sports Health. 2014;6:356-359.

5. Ruiz-Cotorro A, Balius-Matas R, Estruch-Massana AE, Vilaró Angulo J. Spondylolysis in young tennis players. Br J Sports Med. 2006;40:441-446.

6. Sys J, Michielsen J, Bracke P, Martens M, Verstreken J. Nonoperative treatment of active spondylolysis in elite athletes with normal X-ray findings: literature review and results of conservative treatment. Eur Spine J. 2001;10:498-504.

7. Jackson DW, Wiltse LL, Dingeman RD, Hayes M. Stress reactions involving the pars interarticularis in young athletes. $\mathrm{Am} \mathrm{J}$ Sports Med. 1981;9:304-312.

8. Iwamoto J, Takeda T, Wakano K. Returning athletes with severe low back pain and spondylolysis to original sporting activities with conservative treatment. Scand J Med Sci Sports. 2004;14: 346-351.

9. Nozawa S, Shimizu K, Miyamoto K, Tanaka M. Repair of pars interarticularis defect by segmental wire fixation in young athletes with spondylolysis. Am J Sports Med. 2003;31:359-364.

10. Menga EN, Kebaish KM, Jain A, Carrino JA, Sponseller PD. Clinical results and functional outcomes after direct intralaminar screw repair of spondylolysis. Spine (Phila Pa 1976). 2014;39: 104-110.

11. Debnath UK, Freeman BJ, Gregory P, de la Harpe D, Kerslake RW, Webb JK. Clinical outcome and return to sport after the surgical treatment of spondylolysis in young athletes. $J$ Bone Joint Surg Br. 2003;85:244-249.

12. Snyder LA, Shufflebarger H, O'Brien MF, Thind H, Theodore N, Kakarla UK. Spondylolysis outcomes in adolescents after direct screw repair of the pars interarticularis. J Neurosurg Spine. 2014; 21:329-333.

13. Hardcastle PH. Repair of spondylolysis in young fast bowlers. J Bone Joint Surg Br. 1993;75:398-402.

14. Ranawat VS, Dowell JK, Heywood-Waddington MB. Stress fractures of the lumbar pars interarticularis in athletes: a review based on long-term results of 18 professional cricketers. Injury. 2003; 34:915-919.

15. Rossi F, Dragoni S. Lumbar spondylolysis: occurrence in competitive athletes. Updated achievements in a series of 390 cases. J Sports Med Phys Fitness. 1990;30:450-452.

16. Cavalier R, Herman MJ, Cheung EV, Pizzutillo PD. Spondylolysis and spondylolisthesis in children and adolescents: I. Diagnosis, natural history, and nonsurgical management. J Am Acad Orthop Surg. 2006;14:417-424.

17. Anderson K, Sarwark JF, Conway JJ, Logue ES, Schafer MF. Quantitative assessment with SPECT imaging of stress injuries 
of the pars interarticularis and response to bracing. $J$ Pediatr Orthop. 2000;20:28-33.

18. Bell DF, Ehrlich MG, Zaleske DJ, Brace treatment for symptomatic spondylolisthesis. Clin Orthop Relat Res. 1988;(236): 192-198.

19. Blanda J, Bethem D, Moats W, Lew M. Defects of pars interarticularis in athletes: a protocol for nonoperative treatment. J Spinal Disord. 1993;6:406-411.

20. Dutton JA, Hughes SP, Peters AM. SPECT in the management of patients with back pain and spondylolysis. Clin Nucl Med. 2000; 25:93-96.

21. Hensinger RN. Spondylolysis and spondylolisthesis in children and adolescents. J Bone Joint Surg Am. 1989;71:1098-1107.
22. Pizzutillo PD, Hummer CD 3rd. Nonoperative treatment for painful adolescent spondylolysis or spondylolisthesis. J Pediatr Orthop. 1989;9:538-540.

23. Steiner ME, Micheli LJ. Treatment of symptomatic spondylolysis and spondylolisthesis with the modified Boston brace. Spine (Phila Pa 1976). 1985;10:937-943.

24. Deguchi M, Rapoff AJ, Zdeblick TA. Biomechanical comparison of spondylolysis fixation techniques. Spine (Phila Pa 1976). 1999;24:328-333.

25. Noggle JC, Sciubba DM, Samdani AF, Anderson DG, Betz RR, Asghar J. Minimally invasive direct repair of lumbar spondylolysis with a pedicle screw and hook construct. Neurosurg Focus. 2008;25(2):E15. 